\title{
Radiation Through Cylindrical Plasma Sheaths
}

\author{
J. H. Harris
}

\author{
Contribution from the Antenna Department, Hughes Aircraft Company, \\ Culver City, Calif.
}

(Received June 24, 1963)

\begin{abstract}
The radiation fields of a magnetic line source axially oriented on a conducting cylinder which is in a plasma environment have been analytically determined. The plasma environment is taken to be a series of homogeneous cylindrical layers none of which is contiguous with the cylinder. It is demonstrated that a quite significant distortion of the field pattern is effected by the presence of a lossless plasma sheath. Characteristics of the radiation fields are discussed and radiation patterns, computed in a plane through the axis of the cylinder and the line source, are presented. The patterns are found to have large narrow amplitude fluctuations that can be attributed to leaky waves.
\end{abstract}

\section{Introduction}

The gross effects on radio wave propagation of the plasma sheath which envelopes hypersonic vehicles are fairly well understood. It is recognized that when such vehicles travel at sufficient speeds in the upper atmosphere there may be a blackout of radio communication and sufficient distortion of radar signals and patterns to make radar systems unreliable.

The most direct approach to increasing communication and radar system reliability when the sheath environment is present is to increase the propagation frequency well beyond the peak plsama frequency in the shock wave. In this way the sheath is made to appear transparent to the waves so that, aside from an increase in the probability of breakdown on transmit, the sheath might just as well not be there. The problem is, however, that the plasma frequency in some regions of the sheath can go well beyond the frequency of operation of present systems. In regions of the sheath aft of the nose of a vehicle there is a significant decrease in electron density so that, in general, there is a point at which an rf system will operate with only modest distortion of the propagating signals. It is such a region that is of concern in this paper. In particular, we shall investigate the effect of the sheath on propagation when the propagation frequency is greater than the plasma frequency, but when they are both of the same order of magnitude.

The geometrical configuration of the hypersonic plasma sheath in terms of the spatial variation of both charged and uncharged particles has been subjected to a great deal of investigation over the past few years, and while an exact determination of the spatial variation of the constitutive parameters of the sheath has not been made for even the simplest of vehicle configurations, a general description is available for cylindrically symmetrical vehicles with tapered nose sections. In this report the radiation characteristics of a linear slot antenna flush mounted to a circularly cylindrical vehicle will be considered. In the sections 2,3 , and 4 the enveloping plasma sheath will be taken to be a series of consecutive plasma layers contiguous with each other but separated from the body of the vehicle. This separation is characteristic of hypersonic shock waves at other than stagnation regions. The vehicle, as well as the shock wave region, will be taken to be infinitely long and the cylinder will be considered to be infinitely conducting. In section 5 an approximation technique for determining the field distortion due to sheaths which are not necessarily cylindrical and composed of homogeneous layers will be developed and discussed. It will be shown that this technique predicts the significant distortions which have been computed for the single layer case under the assumption of leaky waves excited by the source.

1 This work was sponsored by the Electronics Research Directorate, Air Force Cambridge Research Laboratories, under Contract No. A F 19604-8386. 


\section{Formulation of the Radiation Problem}

\subsection{Solution for the Field in Cylindrical Regions}

The radiation field of a magnetic line source lying on a circularly cylindrical conducting surface is to be determined. The surface is infinite in extent, but the line source is finite and lies parallel to the cylinder axis. Encircling the surface is a series of concentric cylindrical regions containing homogeneous plasma media with arbitrary electron densities, except for the inner and outer regions which have zero electron density. This geometry is depicted in figure 1.

The technique for obtaining a solution to this cylindrical radiation problem is well known. The solution may be found in terms of $E$ and $H$ modes referred to the $z$ direction, which forms the cylinder axis. Expressed in terms of potential functions, $F$ and $A$, satisfying the scalar Helmholtz equation the two sets of modes

$$
\mathbf{E}_{1}=-i \omega \mu \nabla \times\left(F \mathbf{u}_{z}\right) \quad \mathbf{H}_{2}=i \omega \epsilon \nabla \times\left(A \mathbf{u}_{z}\right)
$$

are defined for each cylindrical region. The total field is then

$$
\mathbf{E}=\mathbf{E}_{1}+\frac{1}{i \omega \epsilon} \nabla \times \mathbf{H}_{2} \quad \mathbf{H}=\mathbf{H}_{2}-\frac{1}{i \omega \mu} \nabla \times \mathbf{E}_{1}
$$

The propagation and plasma frequency are considered to be in the kilomegacycle region, so that the cyclotron frequency, which is entirely due to the earth's magnetic field in the absence of sources of low frequency magnetic field on the vehicle, may be neglected. The constitutive parameters in the cylindrical regions are thus isotropic. Further it is assumed that each region is characterized completely by an equivalent constant permittivity.

Solutions for $A$ and $F$ may be given in terms of cylindrical wave functions in the form [Wait, 1959a].

$$
\begin{aligned}
& A=\sum_{m=-\infty}^{\infty} \int_{-\infty}^{\infty} d h\left\{a_{m}(h) H_{m}^{(2)}\left(\sqrt{k^{2}-h^{2}} \rho\right)+A_{m}(h) J_{m}\left(\sqrt{k^{2}-h^{2}} \rho\right)\right\} e^{-i(m \phi+h z)} \\
& F=\sum_{m=-\infty}^{\infty} \int_{-\infty}^{\infty} d h\left\{b_{m}(h) H_{m}^{(2)}\left(\sqrt{k^{2}-h^{2}} \rho\right)+B_{m}(h) J_{m}\left(\sqrt{k^{2}-h^{2}} \rho\right)\right\} e^{-i(m \phi+h z)}
\end{aligned}
$$

and the fields may be found directly from eq (2). The form for the field components transverse

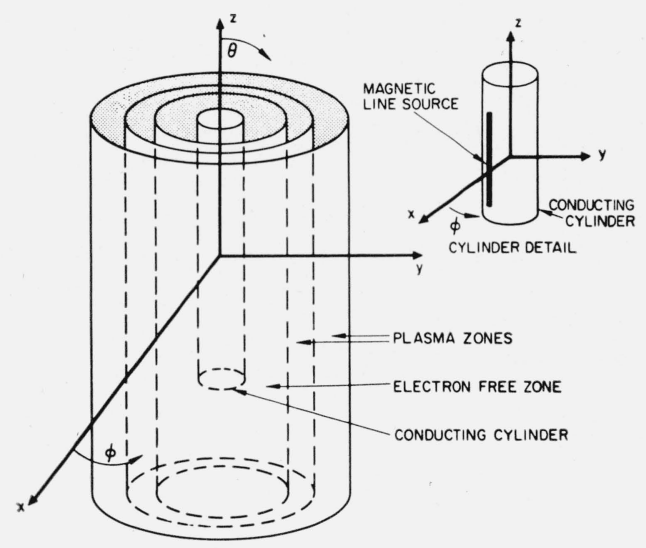

Figure 1. Geometrical configuration for cylindrical plasma sheath problem. 
to the radial direction in any region is

$$
\begin{gathered}
E_{z}=\sum_{m=-\infty}^{\infty} \int_{-\infty}^{\infty} d h\left\{\left(k^{2}-h^{2}\right)\left[a_{m}(h) H_{m}^{(2)}\left(\sqrt{k^{2}-h^{2}} \rho\right)+A_{m}(h) J_{m}\left(\sqrt{k^{2}-h^{2}} \rho\right)\right]\right\} e^{-i(m \phi+h z)} \\
H_{z}=\sum_{m=-\infty}^{\infty} \int_{-\infty}^{\infty} d h\left\{\left(k^{2}-h^{2}\right)\left[b_{m}(h) H_{m}^{(2)}\left(\sqrt{k^{2}-h^{2}} \rho\right)+B_{m}(h) J_{m}\left(\sqrt{k^{2}-h^{2}} \rho\right)\right]\right\} e^{-i(m \phi+h z)} \\
E_{\phi}=\sum_{m=-\infty}^{\infty} \int_{-\infty}^{\infty} d h\left\{i \omega \mu\left(\sqrt{k^{2}-h^{2}}\right)\left[b_{m}(h) H_{m}^{(2) \prime}\left(\sqrt{k^{2}-h^{2}} \rho\right)+B_{m}(h) J_{m}^{\prime}\left(\sqrt{k^{2}-h^{2}} \rho\right)\right]\right. \\
\left.-\frac{m h}{\rho}\left[a_{m}(h) H_{m}^{(2)}\left(\sqrt{k^{2}-h^{2}} \rho\right)+A_{m}(h) J_{m}\left(\sqrt{k^{2}-h^{2}} \rho\right)\right]\right\} e^{-i(m \phi+h z)} \\
H_{\phi}=\sum_{m=-\infty}^{\infty} \int_{-\infty}^{\infty} d h\left\{-\frac{i k^{2}}{\mu \omega}\left(\sqrt{k^{2}-h^{2}}\right)\left[a_{m}(h) H_{m}^{(2)}\left(\sqrt{k^{2}-h^{2}} \rho\right)+A_{m}(h) J_{m}^{\prime}\left(\sqrt{k^{2}-h^{2}} \rho\right)\right]\right. \\
\left.-\frac{m h}{\rho}\left[b_{m}(h) H_{m}^{(2)}\left(\sqrt{k^{2}-h^{2}} \rho\right)+B_{m}(h) J_{m}\left(\sqrt{k^{2}-h^{2}} \rho\right)\right]\right\} e^{-i(m \phi+h z)}
\end{gathered}
$$

Equations (4) represent the complete solution for the field in any homogeneous region of space with characteristic propagation constant $k$. The set of coefficients $a_{m}, A_{m}, b_{m}$, and $B_{m}$ are determined for each region by matching the four field expressions at each boundary. Since the fields are finite in each region the transform operation may be considered to be a factorable operator and the matching technique consists of equating the quantities inside the braces in eq (4).

\subsection{General Formulation for the Multilayer Problem}

To facilitate the writing of solutions the convention adopted will be that the cylindrical surfaces are at radii $\rho_{j}$ where the cylinder surface is $\rho_{0}$ and the outer plasma region is bounded on the outside by $\rho_{N}$. The regions themselves are numbered the same as their outer boundaries so that $k_{j}$ represents the propagation constant of the $j$ th region where $j$ has the values 1,2, . . , $N+1$ and $k_{0}$ is reserved for the plasma free regions, i.e.,

$$
k_{1}=k_{N+1}=k_{0} .
$$

The expansion coefficients of the fields are labeled with a superscript appropriate to their regions in the form $a_{m}^{(j)}$ and as in [Wait, 1959a] a new symbol is defined:

$$
u_{j}=\sqrt{k_{j}^{2}-h^{2}} .
$$

Equating the braces of (4) at the $j$ th boundary yields the four equations

$$
\begin{gathered}
u_{j}^{2}\left[a_{m}^{(j)} H_{m}^{(2)}\left(u_{j} \rho_{j}\right)+A_{m}^{(j)} J_{m}\left(u_{j} \rho_{j}\right)\right]=u_{j+1}^{2}\left[a_{m}^{(j+1)} H_{m}^{(2)}\left(u_{j+1} \rho_{j}\right)+A_{m}^{(j+1)} J_{m}\left(u_{j+1} \rho_{j}\right)\right] \\
u_{j}^{2}\left[b_{m}^{(j)} H_{m}^{(2)}\left(u_{j} \rho_{j}\right)+B_{m}^{(j)} J_{m}\left(u_{j} \rho_{j}\right)\right]=u_{j+1}^{2}\left[b_{m}^{(j+1)} H_{m}^{(2)}\left(u_{j+1} \rho_{j}\right)+B_{m}^{(j+1)} J_{m}\left(u_{j+1} \rho_{j}\right)\right] \\
\begin{array}{c}
i \omega \mu u_{j}\left[b_{m}^{(j)} H_{m}^{(2) \prime}\left(u_{j} \rho_{j}\right)+B_{m}^{(j)} J_{m}^{\prime}\left(u_{j} \rho_{j}\right)\right]-\frac{m h}{\rho_{j}}\left[a_{m}^{(j)} H_{m}^{(2)}\left(u_{j} \rho_{j}\right)+A_{m}^{(j)} J_{m}\left(u_{j} \rho_{j}\right)\right] \\
=i \omega \mu u_{j+1}\left[b_{m}^{(j+1)} H_{m}^{(2) \prime}\left(u_{j+1} \rho_{j}\right)+B_{m}^{(j+1)} J_{m}^{\prime}\left(u_{j+1} \rho_{j}\right)\right] \\
-\frac{m h}{\rho_{j}}\left[a_{m}^{(j+1)} H_{m}^{(2)}\left(u_{j+1} \rho_{j}\right)+A_{m}^{(j+1)} J_{m}\left(u_{j+1} \rho_{j}\right)\right] \\
-\frac{i k_{j}^{2}}{\mu \omega} u_{j}\left[a_{m}^{(j)} H_{m}^{(2) \prime}\left(u_{j} \rho_{j}\right)+A_{m}^{(j)} J_{m}^{\prime}\left(u_{j} \rho_{j}\right)\right]-\frac{m h}{\rho_{j}}\left[b_{m}^{(j)} H_{m}^{(2)}\left(u_{j} \rho_{j}\right)+B_{m}^{(j)} J_{m}\left(u_{j} \rho_{j}\right)\right] \\
=-\frac{i k_{j+1}^{2}}{\mu \omega} u_{j+1}\left[a_{m}^{(j+1)} H_{m}^{(2) \prime}\left(u_{j+1} \rho_{j}\right)+A_{m}^{(j+1)} J_{m}^{\prime}\left(u_{j+1} \rho_{j}\right)\right] \\
-\frac{m h}{\rho_{j}}\left[b_{m}^{(j+1)} H_{m}^{(2)}\left(u_{j+1} \rho_{j}\right)+B_{m}^{(j+1)} J_{m}\left(u_{j+1} \rho_{j}\right)\right] .
\end{array}
\end{gathered}
$$


An analytic solution to the problem is found by determining the $4(N+1)$ modal coefficients $a_{m}^{(j)}, A_{m}^{(j)}, b_{m}^{(j)}, B_{m}^{(j)} ; j=1,2, \ldots, N+1$. Equations (6), when written for the $N$ surfaces, represent $4 N$ equations. The necessary additional four equations are obtained from the radiation condition which requires that there be only outgoing waves in the external region and from matching the fields to the source at the cylinder surface. Thus,

and, at $\rho=\rho_{0}$

$$
A_{m}^{(N+1)}=B_{m}^{(N+1)}=0
$$

$$
\begin{gathered}
E_{z}=\sum_{m=-\infty}^{\infty} \int_{-\infty}^{\infty} d h\left\{u_{1}^{2}\left[a_{m}^{(1)} H_{m}\left(u_{1} \rho_{0}\right)+A_{m}^{(1)} J_{m}\left(u_{1} \rho_{0}\right)\right]\right\} e^{-i(m \phi+h z)}=0 \\
E_{\phi}=\sum_{m=-\infty}^{\infty} \int_{-\infty}^{\infty} d h\left\{i \omega \mu u_{1}\left[b_{m}^{(1)} H_{m}^{(2) \prime}\left(u_{1} \rho_{0}\right)+B_{m}^{(1)} J_{m}^{\prime}\left(u_{1} \rho_{0}\right)\right]\right. \\
=\sum_{m=-\infty}^{\infty} \int_{-\infty}^{\infty} d h\left\{P_{m}(h)\right\} e^{-i(m \phi+h z)} \\
\left.-\frac{m h}{\rho_{0}}\left[a_{m}^{(1)} H_{m}^{(2)}\left(u_{1} \rho_{0}\right)+A_{m}^{(1)} J_{m}\left(u_{1} \rho_{0}\right)\right]\right\} e^{-i(m \phi+h z)}
\end{gathered}
$$

where the expression on the right side of (9) represents the expansion of the line source in a Fourier integral in $z$ and a Fourier series in $\phi$. Equation (9) may then be simplified by equating only the braces.

\subsection{Asymptotic Evaluation of the Radiated Field}

To find the radiated field it is necessary to carry out the integration and summation indicated in (4) when $j=N+1$. The usual technique is to change the cylindrical field coordinates $\rho, \phi$, and $z$ to the spherical coordinates $R, \theta, \phi$ (see fig. 1) and to evaluate the integral asymptotically for large $R$. Thus under the substitutions

$$
\begin{aligned}
& \rho=R \sin \theta \\
& z=R \cos \theta
\end{aligned}
$$

the $\phi$ component of the electric field is

$$
\begin{aligned}
E_{\phi}=\sum_{m=-\infty}^{\infty} \int_{-\infty}^{\infty} d h\left\{i \omega \mu u_{N+1} b_{m}^{(N+1)} H_{m}^{(2) \prime}\left(u_{N+1} R \sin \theta\right)\right. & \\
& \left.-\frac{m h}{R \sin \theta} a_{m}^{(N+1)} H_{m}^{(2)}\left(u_{N+1} R \sin \theta\right)\right\} e^{-i(m \phi+h R \cos \theta) .}
\end{aligned}
$$

For finite $m$ and large value of the argument $u_{N+1} R \sin \theta$, the Hankel function is approximately [Wait, 1959b]

$$
H_{m}^{(2)}\left(u_{N+1} R \sin \theta\right) \approx \sqrt{\frac{2}{\pi u_{N+1} R \sin \theta}} e^{-i\left[u_{N+1} R \sin \theta-\frac{2 m+1}{4} \pi\right]}
$$

and its derivative is

$$
\begin{aligned}
& H_{m}^{(2) \prime}\left(u_{N+1} R \sin \theta\right)=H_{m-1}^{(2)}\left(u_{N+1} R \sin \theta\right)-\frac{m}{u_{N+1} R \sin \theta} H_{m}^{(2)}\left(u_{N+1} R \sin \theta\right) \\
& \approx-i \sqrt{\frac{2}{\pi u_{N+1} R \sin \theta}} e^{-i\left[u_{N+1} R \sin \theta-\frac{2 m+1}{4} \pi\right] .}
\end{aligned}
$$

Substitution of (11) and (12) into (10) yields

$$
\begin{aligned}
E_{\phi}=\sum_{-\infty}^{\infty} \int_{-\infty}^{\infty} d h\left\{\omega \mu u_{N+1} b_{m}^{(N+1)}\right. & \left.-\frac{m h}{R \sin \theta} a_{m}^{(N+1)}\right\} \\
& \times\left\{\sqrt{\frac{2}{\pi u_{N+1} R \sin \theta}} e^{-i\left[u_{N+1} R \sin \theta-\frac{2 m+1}{4} \pi\right]}\right\} e^{-i(m \phi+h R \cos \theta)}
\end{aligned}
$$


which is an integration on the real axis of the $h$-plane. The radiation field is the sum of terms of order $1 / R$. Terms of this order are readily determined from (13) by evaluation of the integral by the method of stationary phase which yields as solution for the integral [Copson, 1946]

$$
I=\int_{x_{0}-\epsilon}^{x_{0}+\epsilon} e^{i R_{\phi}(x)} \psi(x, R) d x
$$

the quantities

$$
I=\left\{\begin{array}{l}
O\left(\frac{1}{R}\right) \psi\left(x_{0}, R\right) \\
\sqrt{\frac{2 \pi}{R \phi^{\prime \prime}\left(x_{0}\right)}} \psi\left(x_{0}, R\right) e^{i\left[R \phi\left(x_{0}\right)+\frac{\pi}{4}\right]}+O\left(\frac{1}{R}\right) \psi\left(x_{0}, R\right)
\end{array}\right.
$$

where the upper solution is obtained if $x_{0}$ is not a stationary point and the lower solution holds when $x_{0}$ is a stationary point. Examination of (13) clearly indicates that the term for which $a_{m}^{(N+1)}$ is a factor yields no radiation fields while the $b_{m}^{(N+1)}$ factor yields a radiation field only at the stationary points of the phase term

which are at

$$
\left(h \cos \theta+u_{N+1} \sin \theta\right)=\left(h \cos \theta+\sqrt{k_{0}^{2}-h^{2}} \sin \theta\right)
$$

$$
h=k_{0} \cos \theta \text {. }
$$

From (16), (15), and (13), then, the radiation field is given by

$$
E_{\phi}=\left.2 i \omega \mu \frac{e^{-i k R}}{R} \sum_{m=-\infty}^{\infty} u_{N+1} b_{m}^{(N+1)}(h) e^{i m\left(\frac{\pi}{2}-\phi\right)}\right|_{h=k_{0} \cos \theta} \dot{ }
$$

Equation (17) represents the $\phi$ component of the radiated field. A similar expression holds for the $\theta$ component of the field so that once the coefficients $b_{m}^{(N+1)}$ and $a_{m}^{(N+1)}$ are determined the field may be computed numerically. Additionally, it bas been shown that the integration over $h$ yields no radiated field except at the stationary points. It should be pointed out, however, that the $h$ integration passes through branch points at $u_{j}=0$ along the real $h$ axis (fig. 2). A plasma sheath differs from a dielectric sheath in that for a plasma the branch points are within the region $-k_{0}<\operatorname{Re} h<k_{0}$, which is the region of integration from which the radiation field is obtained. To remove difficulties that arise because of the presence of the branch points along the integration path, a small loss term may be added to propagation constants in the layers, thereby removing the branch points from the real axis. The branch lines may then be drawn so as not to cross the real axis and the Fourier transform remains valid.

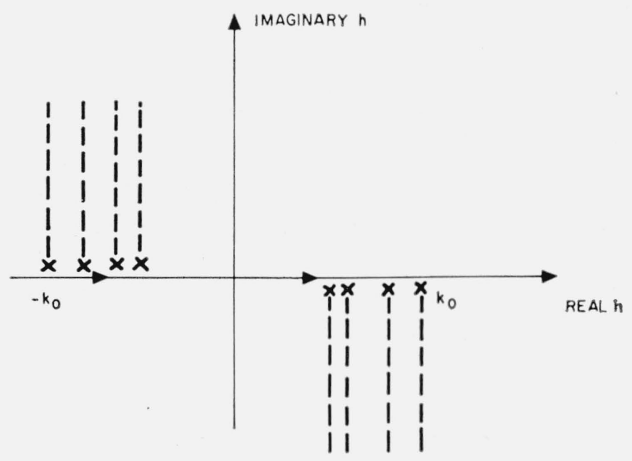

FiguRE 2 Integration path in the h-plane.

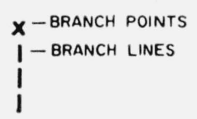




\section{General Characteristics of the Far Field With Several Plasma Layers}

The general solution for the radiation field when several plasma layers are present is difficult to reduce to a useful form because of the size of the expressions and the tedious work required to obtain them. As indicated in section 1, the solution for the field when a single noncontiguous layer is present requires the reduction of 12 linear equations, and this number increases by 4 for every additional layer. Clearly, then, the field expressions are not amenable to an extensive quantitative analysis without resort to numerical solutions obtained with the computer. Nevertheless, some qualitative and semiquantitative information may be gathered about the field pattern without an actual solution of the equations.

\subsection{Polarization of the Field}

The expressions for the radiated field are

$$
\begin{aligned}
& E_{\phi}=\sum_{m=-\infty}^{\infty} \int_{-\infty}^{\infty} d h\left[i \omega \mu u_{0} b_{m}^{(N+1)} H_{m}^{(2) \prime}\left(u_{0} \rho\right)\right] e^{-i(m \phi+h z)} \\
& H_{\phi}=\sum_{m=-\infty}^{\infty} \int_{-\infty}^{\infty} d h\left[\frac{-i k_{0}^{2}}{\mu \omega} u_{0} a_{m}^{(N+1)} H_{m}^{(2)}\left(u_{0} \rho\right)\right] e^{-i(m \phi+h z)}
\end{aligned}
$$

where $E_{\phi}$ and $H_{\phi}$ represent the electric and magnetic field vectors respectively of the orthogonal components of the radiated field. It will be shown in the following section that

$$
\begin{aligned}
& a_{m}^{(N+1)} H_{m}^{(2)}\left(u_{0} \rho\right) \text { is odd in } m \text { and } \\
& b_{m}^{(N+1)} H_{m}^{(2)}\left(u_{0} \rho\right) \text { is even in } m,
\end{aligned}
$$

and since the derivatives of the Hankel functions have the same behavior in $m$ as the Hankel functions themselves, eq (18) can be written in the form

where

$$
\begin{aligned}
& E_{\phi}=\sum_{0}^{\infty} \frac{e_{m}}{2} \int_{-\infty}^{\infty} d h\left[i \omega \mu u_{0} b_{m}^{(N+1)} H_{m}^{(2) \prime}\left(u_{0} \rho\right)\right] e^{-i h z}\left[e^{-i m \phi}+e^{i m \phi}\right] \\
& H_{\phi}=\sum_{0}^{\infty} \frac{e_{m}}{2} \int_{-\infty}^{\infty} d h\left[-\frac{i k_{0}^{2}}{\mu \omega} u_{0} a_{m}^{(N+1)} H_{m}^{(2) \prime}\left(u_{0} \rho\right)\right] e^{-i h z}\left[e^{-i m \phi}-e^{i m \phi}\right]
\end{aligned}
$$

$$
e_{m}=\left\{\begin{array}{l}
1 m=0 \\
2 m>0 .
\end{array}\right.
$$

The field expressions in (19) indicate that the radiated field is in general elliptically polarized with $E_{\phi}$ given by the upper expression in eq (19) and $E_{\phi}$ given by

$$
E_{\theta}=\sqrt{\frac{\mu_{0}}{\epsilon_{0}}} H_{\phi}
$$

where $H_{\phi}$ is the lower expression in (19). In the planes $\phi=0$ and $\phi=\pi$, the $E_{\theta}$ component is seen to be zero and the field is plane polarized. Elsewhere, both components of the field are present so that the plasma sheath is seen to introduce a transverse electric field component that is not present without the sheath. The order of magnitude of this transverse field, which determines the extent of the ellipticity of the radiated field, is difficult to ascertain without actual numerical computations, but it is expected that the degree of ellipticity will be small.

\subsection{Proof of the Even and Odd Nature of the Modal Coefficients}

In order to symbolically depict the $4(N+1)$ equations needed to determine the modal coefficients, figure 3 is generated, which may be thought of as a representation of the matrix that multiplies the line matrix of unknown coefficients to yield the source function $P_{m}(h)$. The shaded areas in the illustration are the nonzero sections of the matrix, although particular 


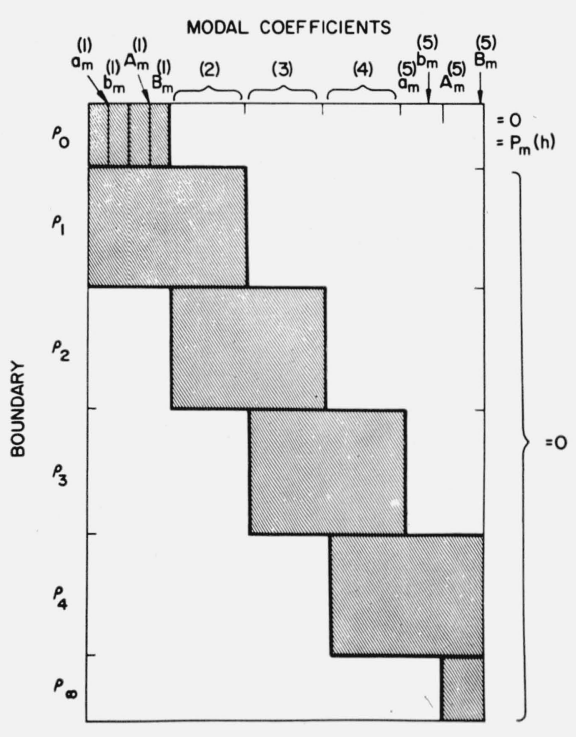

Figure 3. Symbolical representation of $4(\mathrm{~N}+1)$ linear boundary matching equations for $\mathrm{N}=4$.

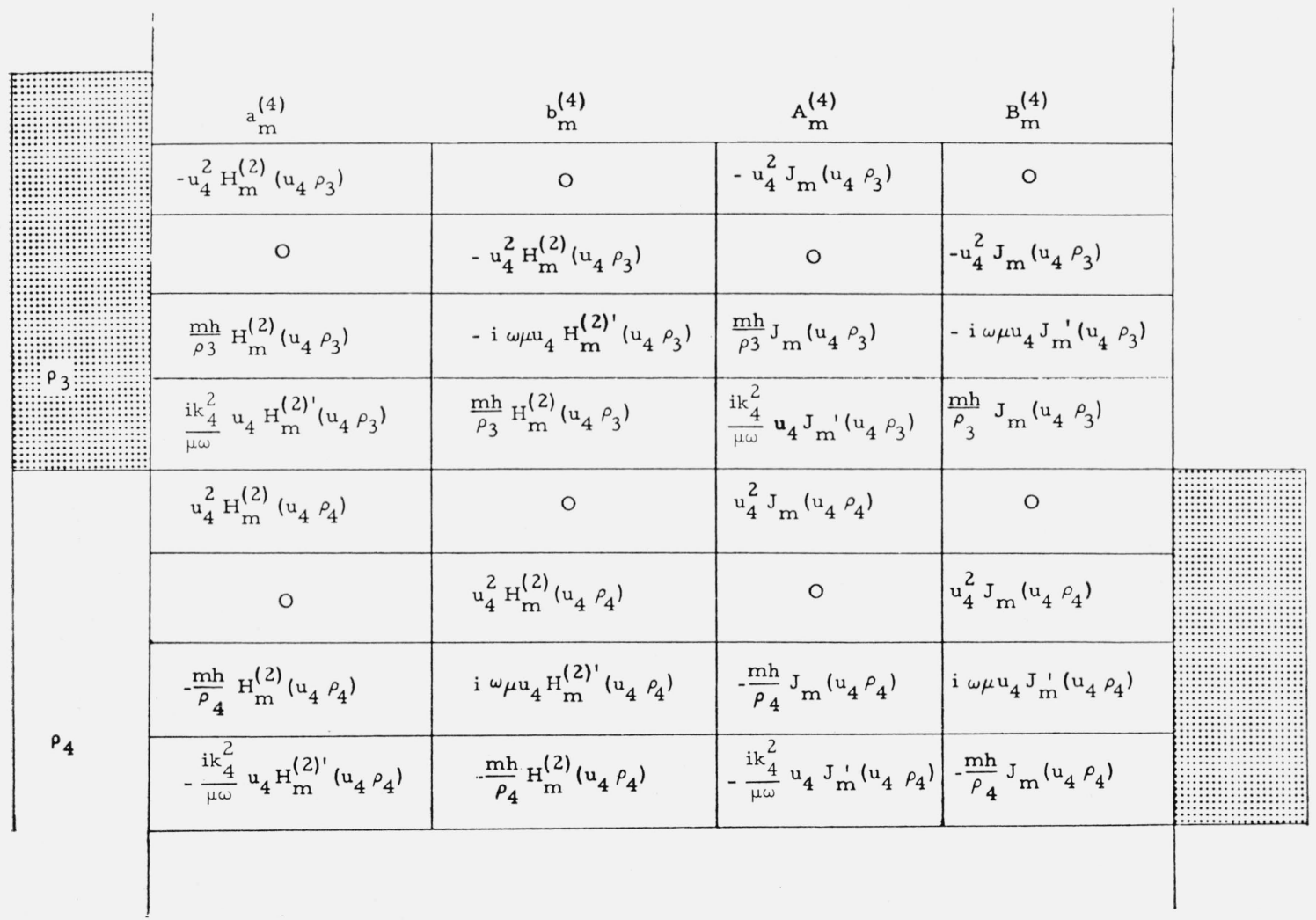

FIgURE 4. Detail of a typical 4-column from figure 3 showing the multipliers of the modal coefficients for region 4 at the boundaries $\rho_{3}$ and $\rho_{4}$.

coefficients within the shaded section are zero as is indicated in figure 4 which is a detail of the shaded region of a typical 4-column of figure 3 .

It may be seen from figures 3 and 4 that the $m$ dependent terms required to compute $a_{m}^{(N+1)}$ and $b_{m}^{(N+1)}$ are the Bessel and Hankel functions and their first derivatives and the quantity $m$ itself. The function $P_{m}(h)$ is independent of $m$ for the line source. In view of this the notation below is used

$$
\begin{aligned}
& a_{m}^{(N+1)}=f\left(m, H_{m}, J_{m}\right) \\
& b_{m}^{(N+1)}=g\left(m, H_{m}, J_{m}\right) .
\end{aligned}
$$


First it will be shown that

then that

$$
\begin{aligned}
& f\left(m, H_{-m}, J_{-m}\right)=(-1)^{-m} f\left(m, H_{m}, J_{m}\right) \\
& g\left(m, H_{-m}, J_{-m}\right)=(-1)^{-m} g\left(m, H_{m}, J_{m}\right)
\end{aligned}
$$

$$
\begin{aligned}
& f\left(-m, H_{m}, J_{m}\right)=-f\left(m, H_{m}, J_{m}\right) \\
& g\left(-m, H_{m}, J_{m}\right)=g\left(m, H_{m}, J_{m}\right)
\end{aligned}
$$

and finally that

$$
\begin{aligned}
& a_{m}^{(N+1)} H_{m}^{(2)}\left(u_{0} \rho\right) \text { is odd in } m \\
& b_{m}^{(N+1)} H_{m}^{(2)}\left(u_{0} \rho\right) \text { is even in } m .
\end{aligned}
$$

Consider the equations depicted in figure 3. A solution may be found for $a_{m}^{(N+1)}$ and $b_{m}^{(N+1)}$ by taking the quotient of the appropriate determinants. Every term in the determinant in the denominator will contain either a cylinder function or a zero. The determinant in the numerator will be missing a column containing cylinder functions since the forcing function will replace the appropriate column. The Bessel and Hankel functions and their derivatives behave as [Sommerfeld, 1949]

so that

$$
\begin{aligned}
& H_{-m}^{(2)}(x)=(-1)^{m} H_{m}^{(2)}(x) \\
& J_{-m}(x)=(-1)^{m} J_{m}(x)
\end{aligned}
$$

$$
\begin{aligned}
& a_{-m}^{(N+1)}=f\left(-m, H_{-m}, J_{-m}\right)=f\left(-m,(-1)^{m} H_{m},(-1)^{m} J_{m}\right) \\
& b_{-m}^{(N+1)}=g\left(-m, H_{-m}, J_{-m}\right)=g\left(-m,(-1)^{m} H_{m},(-1)^{m} J_{m}\right) .
\end{aligned}
$$

Now, a number common to a column of a determinant may be factored from the determinant without changing the value of the determinant. If all the $H_{-m}^{(2)}(x)$ and $J_{-m}(x)$ in the expressions for $a_{-m}^{(N+1)}$ and $b_{-m}^{(N+1)}$ are replaced by the equivalent terms given in eq (25) and then the factors $(-1)^{m}$ are canceled from corresponding columns in the determinants in the numerator and denominator, the result will be that all such $(-1)^{m}$ terms will cancel but one, left over in the denominator. Hence,

$$
\begin{aligned}
& f\left(m, H_{-m}, J_{-m}\right)=(-1)^{-m} f\left(m, H_{m}, J_{m}\right) \\
& g\left(m, H_{-m}, J_{-m}\right)=(-1)^{-m} g\left(m, H_{m}, J_{m}\right) .
\end{aligned}
$$

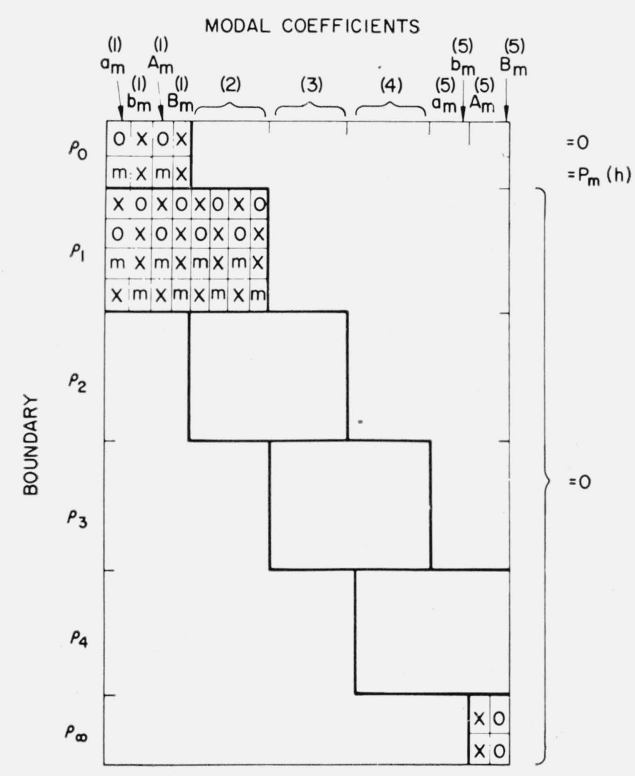

Figure 5. Symbolical representation of $4(\mathrm{~N}+1)$ linear boundary matching equations for $\mathrm{N}=4$ with zeros, $\mathrm{m}$, and non $\mathrm{m}$ coefficients placed in evidence. 
Now, to find the behavior of $a_{m}^{(N+1)}$ and $b_{m}^{(N+1)}$ with $m$ itself, once again consider figure 3 . If that figure is reconstructed so that zero coefficients, coefficients containing $m$, and coefficients not containing $m$ are placed in evidence, the symbolic representation of figure 5 is obtained for the boundary matching equations. Clearly the $4(N+1)$ equations can be reduced by eliminating one variable at a step starting with $a_{m}^{(1)}$ on the left side of figure 3 . Examination of the continued application of this process reveals that coefficients that are even multiples of $m$ are always added to even multiples of $m$ and vice versa and, furthermore, that the forcing function will always have factors that are of the same even or odd nature as the factors which multiply $b_{m}^{(j)}$ and $B_{m}^{(j)}$. If the process of reducing one variable at a time is continued until the solution for $b_{m}^{(N+1)}$ is found, it is clear that $b_{m}^{(N+1)}$ has the same even or odd character as $P_{m}(h)$, which is even in $m$, and $a_{m}^{(N+1)}$ has an odd behavior in $m$. Thus

or

$$
\begin{aligned}
& f\left(-m, H_{m}, J_{m}\right)=-f\left(m, H_{m}, J_{m}\right) \\
& g\left(-m, H_{m}, J_{m}\right)=g\left(m, H_{m}, J_{m}\right)
\end{aligned}
$$

$$
\begin{aligned}
& f\left(-m, H_{-m}, J_{-m}\right)=-f\left(m, H_{-m}, J_{-m}\right) \\
& g\left(-m, H_{-m}, J_{-m}\right)=g\left(m, H_{-m}, J_{-m}\right) .
\end{aligned}
$$

On taking eqs (27) and (29) together, it is found that

$$
\begin{aligned}
& f\left(-m, H_{-m}, J_{-m}\right)=-(-1)^{-m} f\left(m, H_{m}, J_{m}\right) \\
& g\left(-m, H_{-m}, J_{-m}\right)=(-1)^{-m} g\left(m, H_{m}, J_{m}\right) .
\end{aligned}
$$

If the expressions in eq (30) are multiplied by $H_{m}^{(2)}\left(u_{0} \rho\right)$, which has the $m$ behavior indicated in eq $(25)$, the desired result is found:

$$
\begin{aligned}
& a_{-m}^{(N+1)} H_{-m}^{(2)}\left(u_{0} \rho\right)=-a_{m}^{(N+1)} H_{m}^{(2)}\left(u_{0} \rho\right) \\
& b_{-m}^{(N+1)} H_{-m}^{(2)}\left(u_{0} \rho\right)=b_{m}^{(N+1)} H_{m}^{(2)}\left(u_{0} \rho\right) .
\end{aligned}
$$

\subsection{Critical Angles of the Field}

The radiation field in the direction $\theta$ was shown to be contributed by the integration along the infinitesimal portion of the real $h$ axis about the point

$$
h=k_{0} \cos \theta .
$$

Additionally, it was shown that singularities at the points

$$
h=k_{j}
$$

could be removed from the real $h$ axis with the addition of a small loss term to the propagation constants in the layers. The contribution to the radiation field of these singular points take place in the directions

$$
\cos \theta= \pm k_{j} / k_{0}
$$

which are exactly the critical angles defined in ray optics at which total reflection occurs. For angles of ray incidence beyond the critical angle ray optics predicts no transmission. An exact solution to the reflection problem of a plane wave incident at greater than the critical angle on an infinite plane plasma slab of finite thickness, however, indicates that power is coupled through the slab. The amount of power coupled decreases with increasing angle of incidence beyond the critical angle and drops off at a faster rate as the electron density and the thickness of the slab are increased.

For the cylinder problem the solution may be found explicitly for regions defined by angles within and beyond the critical angles of the various layers. Within the first critical angles, the field considered as a function of $\theta$ depends on the behavior of the various Hankel 
and Bessel functions of real argument. When the critical angle is reached, in an imaginary traversal of the $\phi=0$ plane through angles in $\theta$ starting at $90^{\circ}$, the arguments of some of the Hankel and Bessel functions become imaginary. Interpolating from the behavior of plane waves propagating through slabs, the radiated fields are non-zero at the critical angles and decre ase outside the zone defined by these critical angles. In section 4 some computed radiation field configurations are shown, but these computations were limited to the region within the first critical angles because of the added computational difficulties involved in going beyond this point. Thus an actual determination of the fields in the region beyond the first critical angle for the parameter values considered must await further computations. To aid in visualizing the above comments, some typical critical angles are shown in figure 6 .

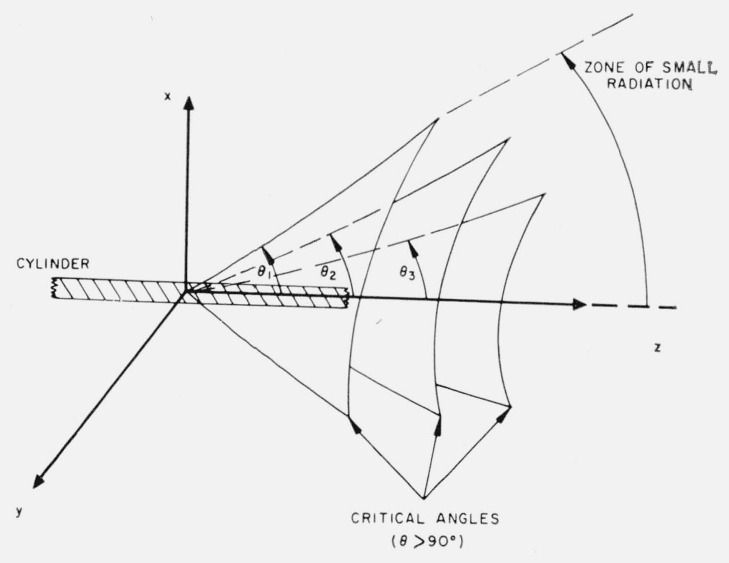

Figure 6. Critical angles of the field for a threelayer plasma.

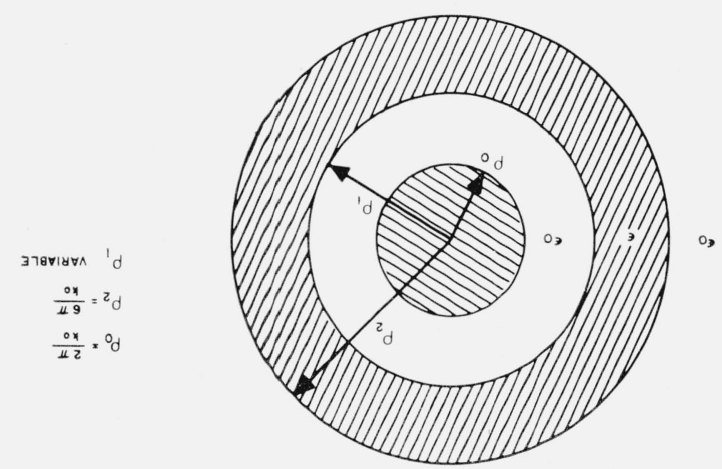

Figure 7. Cylinder encircled by a single plasma layer.

\section{Exact Solution for the Single Plasma Layer Problem}

In this section the radiation pattern of an axial magnetic line source on a conducting cylinder is considered when a single homogeneous plasma layer which is not contiguous with the cylinder is present. This geometrical configuration is of importance because of its obvious relation to the problem of propagation through the boundary layer region of the hypersonic shock wave.

From the discussion in section 2 the solution of this problem involves the solution of 12 linear equations, or actually of 10 nontrivial equations if $A_{m}^{(3)}$ and $B_{m}^{(3)}$ are excluded in advance. The solution for $b_{m}^{(3)}(h)$ was found, but due to its length only the computed results are presented.

The expression for the radiation field as given in (17) may be factored into two parts in the form

$$
E_{\phi}=E_{\phi}^{0} \int_{\text {Aperture }} V(z) e^{i k_{0} z \cos \theta} d z
$$

where $E_{\phi}^{0}$ is the field of a point source on the cylinder and the other factor includes the element and array factors. A proper terminology for $E_{\phi}^{0}$ is thus "cylinder factor." This cylinder factor was programed for computation on the IBM 7090 for the case of a single plasma layer present and for the plasmaless case. ${ }^{2}$ The program was established with the electron density and cylinder and plasma radii on separate data cards so that these parameters could be changed readily. (See fig. 7.)

As stated above, the solution for $b_{m}^{(3)}$ is quite lengthy and complicated, as is the machine program used to compute the field. In order to develop an independent check on both, a set of solutions for the plasma case was taken for decreasing values of electron density and, indeed,

2 The field with o plasma present is given by [Wait, 1959c]. 
the field was found to converge to the plasmaless case. The minimum value of electron density taken was that corresponding to a relative permittivity of 0.99 . The convergence properties of the series were also examined through separate print outs and found to be quite good. The field generally converged to five figure accuracy in about 14 terms where the outer plasma radius, yielding the largest cylinder function arguments, was kept constant at

$$
\rho_{2}=\frac{6 \pi}{k_{0}}
$$

Computations were made in the plane of the line source and the axis of the cylinder, and results are presented in figures $8 \mathrm{a}$ through $8 \mathrm{~g}$. To insure computational accuracy, fairly modest values for the radii were chosen. The cylinder radius was kept constant at

$$
\rho_{0}=\frac{2 \pi}{k_{0}}
$$

the outer plasma radius as above, and the inner plasma radius was taken at $\frac{3.5 \pi}{k_{0}}$ and $\frac{4 \pi}{k_{0}}$, a difference of a quarter free space wavelength. The electron densities correspond to

$$
\frac{\epsilon}{\epsilon_{0}}=0.40,0.80,0.90,0.95, \text { and } 0.99
$$

The chief characteristic of the figures is the extremely sharp variations in field magnitude at certain angles. In order not to miss the sharp fluctuations, the field was computed at $1 / 4^{\circ}$ intervals for the $\frac{\epsilon}{\epsilon_{0}}=0.4$ and 0.8 cases, and even here the mesh was often not fine enough. In figure $8 \mathrm{c}\left(\frac{\epsilon}{\epsilon_{0}}=0.8, \rho_{1}=\frac{3.5 \pi}{k_{0}}, \theta=42.5^{\circ}\right)$ the field rises to a peak $25 \mathrm{db}$ above the free space field in a beam only $1 / 2^{\circ}$ wide. This beam and other beams were checked at $1 / 10^{\circ}$ intervals, but in most cases a simple graphical interpolation was used to complete the curve between $1 / 4^{\circ}$ intervals. The presence of these sharp beams implies the existence of leaky waves that travel down the cylindrical, lossless, infinite structure and produce highly directive beams which add to the other fields. The nature of the radiation field of leaky waves traveling along such a structure and the effect which might be expected if the plasma were lossy and of finite length are discussed in the next section.

A second characteristic of the patterns is that the field variations take place around an average which is approximately the plasmaless field. The total power radiated in the region between the critical angles appears to be about the same whether the plasma is present or not and the field near broadside remains fairly constant for the electron densities considered here. A low-power antenna with a narrow broadside pattern might be expected to operate fairly well in such an environment, but wide and/or scanned beams would be severely distorted.

\section{An Approximation Technique for Quasi-Cylindrical Problems}

It was pointed out in the introduction that the aspect of the plasma sheath problem which is of practical interest for mapping and related applications is the one in which the coupling between the rf energy and the sheath is fairly light; that is, the case in which the maximum relative permittivity in the sheath does not differ by very much from unity. This aspect of the problem suggests that the general class of approximation techniques developed from integral equation solutions for the field are applicable.

In the following paragraphs an exact integral expression will be developed for the field and the feasibility of approximating the solution to problems related to the cylindrical plasma sheath problem will be examined. 


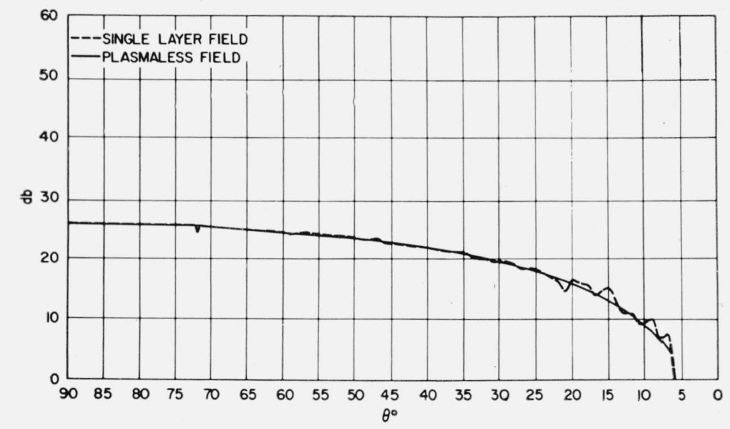

FIGURE 8a. Sheath relative permittivity $=0.99 ;$ sheath inner radius $=3.5 \pi / \mathrm{k}_{0}$.

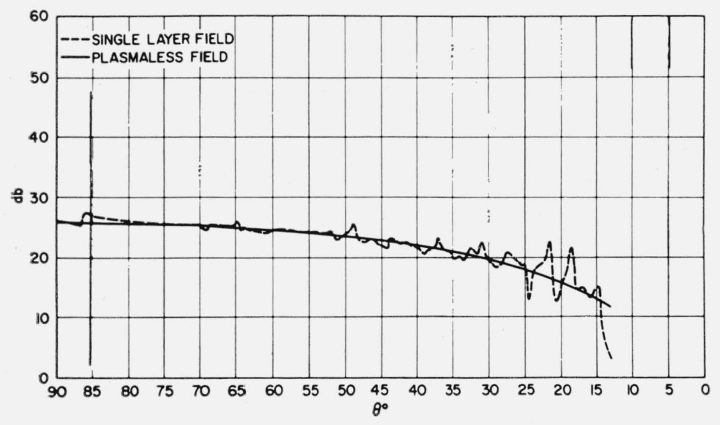

Figure 8b. Sheath relative permittivity $=0.95$; sheath inner radius $=3.5 \pi / \mathrm{k}_{0}$.

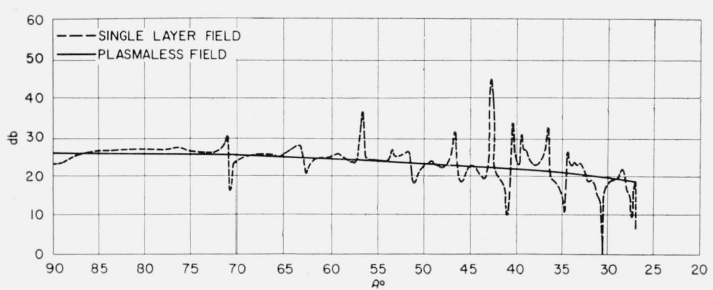

Figure 8d. Sheath relative permittivity $=0.80$; sheath inner radius $=3.5 \pi / \mathrm{k}_{0}$.

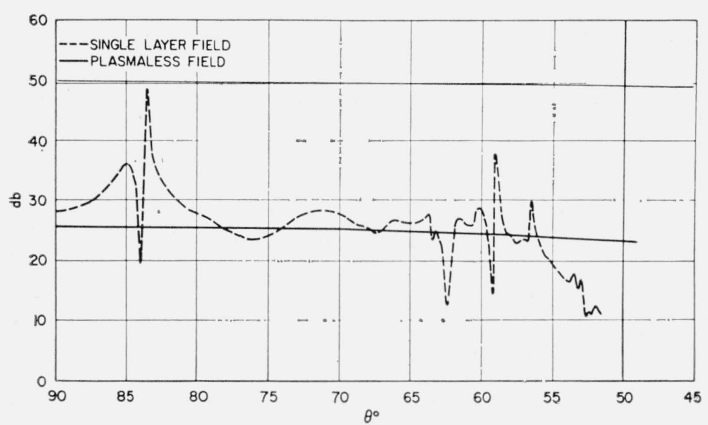

FIGURE 8f. Sheath relative permittivity $=0.40$; sheath inner radius $=3.5 \pi / \mathrm{k}_{0}$.

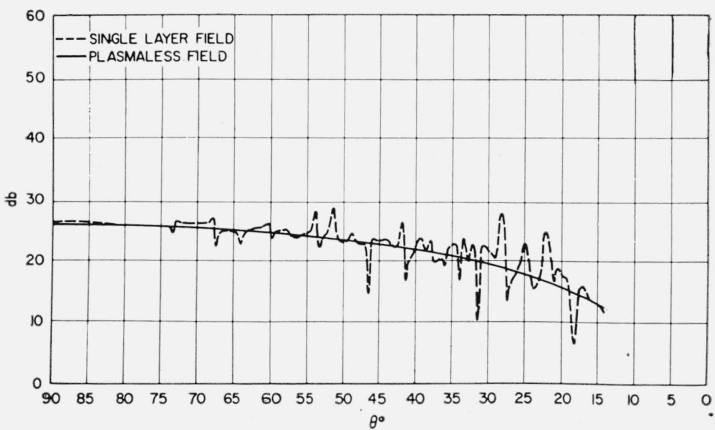

FIGURE 8c. Sheath relative permittivity $=0.90$; sheath inner radius $=3.5 \pi / \mathrm{k}_{0}$

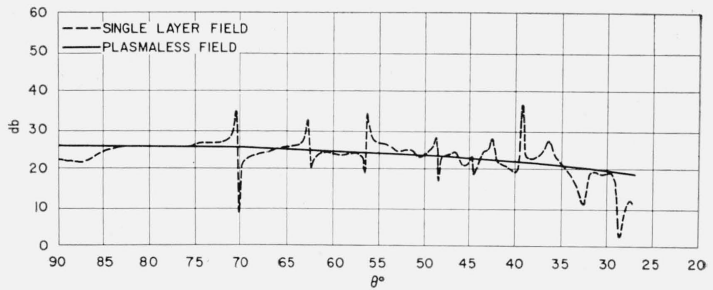

FIGURE 8e. Sheath relative permittivity $=0.80$; sheath inner radius $=4 \pi / \mathrm{k}_{0}$.

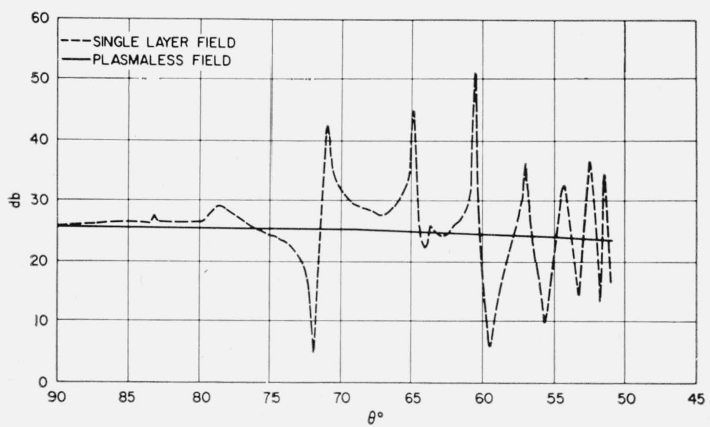

FIGURE $8 \mathrm{~g}$. Sheath relative permittivity $=0.40$; sheath inner radius $=4 \pi / \mathrm{k}_{0}$.

FIGURE 8. Radiation fields through a single-layer cylindrical plasma sheath computed in the plane of the axis of the cylinder and the line source. 


\subsection{Development of the Approximate Field Expression}

Maxwell's equations in isotropic media, in which $\epsilon$ is a continuous function of space, are

$$
\begin{array}{llll}
\nabla \times \mathbf{E}=-j \omega \mu \mathbf{H} & \text { (a) } & \nabla \cdot \epsilon \mathbf{E}=0 & \text { (c) } \\
\nabla \times \mathbf{H}=j \omega \epsilon \mathbf{E} & \text { (b) } & \nabla \cdot \mathbf{H}=0 . & \text { (d) }
\end{array}
$$

The curl of (36a) is taken and curl $\mathbf{H}$ substituted from (36b). This results in

$$
\nabla \times \nabla \times \mathbf{E}=\omega^{2} \mu \epsilon \mathbf{E}
$$

When the vector identities

$$
\nabla \times \nabla \times \mathbf{E}=\nabla(\nabla \cdot \mathbf{E})-\nabla^{2} \mathbf{E}
$$

and

$$
\nabla \cdot \epsilon \mathbf{E}=\epsilon \nabla \cdot \mathbf{E}+(\nabla \epsilon) \cdot \mathbf{E}
$$

are employed, (38) reduces to

$$
\left(\nabla^{2}+k^{2}\right) \mathbf{E}=-\nabla \frac{1}{\epsilon}(\nabla \epsilon \cdot \mathbf{E})
$$

where

$$
k^{2}=\omega^{2} \mu \epsilon .
$$

Now a scalar Green's function is defined; it satisfies the scalar Helmholtz equation with a point source inhomogeneity, i.e.,

$$
\left(\nabla^{2}+k_{0}^{2}\right) G\left(\mathbf{r}, \mathbf{r}^{\prime}\right)=\delta\left(\mathbf{r}-\mathbf{r}^{\prime}\right)
$$

where $\mathbf{r}$ and $\mathbf{r}^{\prime}$ are coordinate vectors. To obtain an integral expression (39) is multiplied by $G$, and (41) by E. These are then subtracted, integrated over space, and the coordinate variables are reversed to obtain

$$
E(\mathbf{r})=\int\left(\mathbf{E} \nabla^{2} G-G \nabla^{2} \mathbf{E}\right) d v^{\prime}-\int G \nabla \frac{1}{\epsilon}(\nabla \epsilon \cdot \mathbf{E}) d v^{\prime}+\int\left(k_{0}^{2}-k^{2}\right) G \mathbf{E} d v^{\prime} .
$$

The first term in (42) may be further reduced to a surface integral by applying the scalar Green's theorem. Additionally, if a Green's function is selected so that it vanishes on the cylinder surface, eq (42) becomes

$$
E(\mathbf{r})=\int \mathbf{E}(\nabla G \cdot d s)+\int\left(k_{0}^{2}-k^{2}\right) G \mathbf{E} d v^{\prime}-\int G \nabla \frac{1}{\epsilon}(\nabla \epsilon \cdot \mathbf{E}) d v^{\prime} .
$$

Equation (43) is an integral equation for the electric field which has little utility when an exact solution for the field is required. Even for the case in which $\epsilon=\epsilon_{0}$ throughout the region external to the conducting cylinder, i.e., when the second and third terms in (43) are zero, a direct solution cannot readily be found because the surface integral in (43) must be taken over the radial component of the field on the cylinder as well as the source field in the aperture. Nevertheless, a good approximation to the radiated field may be found by taking the surface integral over the aperture alone [Silver, 1949]. This approximation is especially true in regions near broadside.

For the inhomogeneous case the effect of the plasma on the radiation field within the critical angles is a perturbation on the fields when no plasma is present. Further, the first term in (43) represents the plasmaless field. The error in this assumption is once again neglect of the radial field at the cylinder, which is altered by the presence of the plasma. The second and third terms in (43) thus are taken to represent the field perturbation due to the plasma and, in fact, the third term will be neglected; it is essentially an integration over the localized charge density in the plasma. That is, the third term is considered a less significant term than the second which represents an integration over the currents in the plasma. 
The above comments are represented by (44) and (45)

$$
\begin{aligned}
& \mathbf{E}_{\text {tota } 1} \approx \mathbf{E}_{\text {no pl asma }}+\mathbf{E}_{\text {pl asma }} \\
& \mathbf{E}_{\text {pl asma }} \approx \mathcal{S}\left(k_{0}^{2}-k^{2}\right) G \mathbf{E} d v^{\prime}
\end{aligned}
$$

\subsection{Integration Over the Plasma}

The scalar Green's function defined by (41) and the condition that $G=0$ at the cylinder is [Wait, 1959c]

$$
G=\frac{i}{8 \pi} \sum_{m=-\infty}^{\infty} \int_{-\infty}^{\infty} d h H_{m}\left(u_{0} \rho\right)\left[J_{m}\left(u_{0} \rho^{\prime}\right)-H_{m}^{(2)}\left(u_{0} \rho^{\prime}\right) \frac{J_{m}\left(u_{0} \rho_{0}\right)}{H_{m}^{(2)}\left(u_{0} \rho_{0}\right)}\right] e^{-i\left[m\left(\phi-\phi^{\prime}\right)+h\left(z-z^{\prime}\right)\right]}
$$

where the primed coordinates refer to the space containing the plasma and the unprimed coordinates refer to the field space. To find any particular far field component the function $\left(k_{0}^{2}-k^{2}\right) G$ is multiplied by the components of $\mathbf{E}$ in the plasma parallel to the far field vector. For example, to find the $\phi$ component of electric field radiated by the plasma

$$
E_{\phi_{\text {plasma }}}=\int_{\text {plasma }}\left(k_{0}^{2}-k^{2}\right) G \mathbf{E}\left(\mathbf{r}^{\prime}\right) \cdot \mathbf{e}_{\phi} d v^{\prime}
$$

where $\mathbf{e}_{\phi}$ is the unit vector parallel to the far field vector. Of special interest is the plane $\phi=0$. In this plane, (47) and hence the field radiated by the currents in the plasma reduces to

$$
E_{\phi_{\text {plasma }}}=\int_{\text {plasma }}\left(k_{0}^{2}-k^{2}\right) G\left[\cos \phi^{\prime} E_{\phi^{\prime}}+\sin \phi^{\prime} E_{\rho^{\prime}}\right] d v^{\prime} .
$$

Now, the term inside the square brackets in (48) may, in general, be expanded in a Fourier series in $\phi^{\prime}$ and a Fourier integral in $z^{\prime}$ to yield

$$
\left[\cos \phi^{\prime} E_{\phi^{\prime}}+\sin \phi^{\prime} E_{\rho^{\prime}}\right]=\sum_{-\infty}^{\infty} \int_{-\infty}^{\infty} d l \alpha_{n}\left(l, \rho^{\prime}\right) e^{-i\left(n \phi^{\prime}+l z^{\prime}\right)}
$$

where $n$ and $l$ are the transform variables. This form for the field is of course the same form as was employed previously for the exact solution to the cylinder problem. Substitution of (49) and (46) into (48) results in

$$
\begin{aligned}
& E_{\phi_{\text {plasma }}}=\int_{-\infty}^{\infty} d z^{\prime} \int_{\rho_{0}}^{\infty} \rho^{\prime} d \rho^{\prime} \int_{0}^{2 \pi} d \phi^{\prime} \sum_{m=-\infty}^{\infty} \sum_{n=-\infty}^{\infty} \int_{-\infty}^{\infty} d h \int_{-\infty}^{\infty} d l \\
& \cdot\left\{\left(k_{0}^{2}-k^{2}\right) e^{-i\left[-m \phi^{\prime}+h\left(z-z^{\prime}\right)\right]} H_{m}^{(2)}\left(u_{0} \rho\right)\left[J_{m}\left(u_{0} \rho^{\prime}\right)-H_{m}^{(2)}\left(u_{0} \rho^{\prime}\right) \frac{J_{m}\left(u_{0} \rho_{0}\right)}{H_{m}^{(2)}\left(u_{0} \rho_{0}\right)}\right] \cdot \alpha_{n}\left(l, \rho^{\prime}\right) e^{-i\left(n \phi^{\prime}+l z^{\prime}\right)}\right\} .
\end{aligned}
$$

A cylindrically symmetrical plasma sheath has been assumed so that the integration on $\phi^{\prime}$ may be carried out directly to yield

$$
\begin{aligned}
E_{\phi_{\text {plasma }}}=2 \pi & \int_{-\infty}^{\infty} d z^{\prime} \int_{\rho_{0}}^{\infty} \rho^{\prime} d_{\rho^{\prime}} \sum_{m=-\infty}^{\infty} \int_{-\infty}^{\infty} d h \int_{-\infty}^{\infty} d l \\
& \cdot\left\{\left(k_{0}^{2}-k^{2}\right) e^{-i n\left(z-z^{\prime}\right)} H_{m}^{(2)}\left(u_{0} \rho\right)\left[J_{m}\left(u_{0} \rho^{\prime}\right)-H_{m}^{(2)}\left(u_{0} \rho^{\prime}\right) \frac{J_{m}\left(u_{0} \rho_{0}\right)}{H_{m}^{(2)}\left(u_{0} \rho_{0}\right)}\right] \alpha_{m}\left(l, \rho^{\prime}\right) e^{-i l z^{\prime}}\right\} .
\end{aligned}
$$

In a similar manner the integration on $z^{\prime}$ may be carried out, but here there is the possibility that $\left(k_{0}^{2}-k^{2}\right)$ varies with $z^{\prime}$. In that event the $z^{\prime}$ integration yields the Fourier transform of $\left(k_{0}^{2}-k^{2}\right)$ in the transform variable $(h-l)$. The integration over $l$ will then yield the convolution of the transform of $\left(k_{0}^{2}-k^{2}\right)$ with $\alpha_{m}\left(l, \rho^{\prime}\right)$, which is denoted by $\beta_{m}\left(h, \rho^{\prime}\right)$. Then (51) reduces to 


$$
\begin{aligned}
E_{\phi_{\text {plasma }}}=2 \pi \int_{\rho_{0}}^{\infty} \rho^{\prime} d \rho^{\prime} \sum_{m=-\infty}^{\infty} \int_{-\infty}^{\infty} d h \\
\cdot\left\{\beta_{m}\left(h, \rho^{\prime}\right) e^{-i h z} H_{m}^{(2)}\left(u_{0} \rho\right)\left[J_{m}\left(u_{0} \rho^{\prime}\right)-H_{m}^{(2)}\left(u_{0} \rho^{\prime}\right) \frac{J_{m}\left(u_{0} \rho_{0}\right)}{H_{m}^{(2)}\left(u_{0} \rho_{0}\right)}\right]\right\} .
\end{aligned}
$$

The integration on $h$ may be carried out for large $\rho$ as in (17) to yield

$$
E_{\phi_{\text {plasma }}}=-4 \pi \frac{e^{-i k R}}{R} \int_{\rho_{0}}^{\infty} \rho^{\prime} d \rho^{\prime} \sum_{m=-\infty}^{\infty}\left\{\beta_{m}\left(h, \rho^{\prime}\right) e^{i m \pi / 2} \cdot\left[J_{m}\left(u_{0} \rho^{\prime}\right)-H_{m}^{(2)}\left(u_{0} \rho^{\prime}\right) \frac{J_{m}\left(u_{0} \rho_{0}\right)}{H_{m}^{(2)}\left(u_{0} \rho_{0}\right)}\right]\right\}_{h=k_{0} \cos \theta} .
$$

\subsection{Characteristics of the Radiation Fields of the Plasma Currents}

Application of (53) to the approximate solution of the type of cylindrical and quasicylindrical problems of concern here is by no means simple. The prime source of difficulty lies in the extremely limited class of functions $\beta_{m}\left(h, \rho^{\prime}\right)$ [Sonine, 1880] which, when a factor in the integrand

$$
\rho^{\prime} \beta_{m}\left(h, \rho^{\prime}\right)\left[J_{m}\left(u_{0} \rho^{\prime}\right)-H_{m}^{(2)}\left(u_{0} \rho^{\prime}\right) \frac{J_{m}\left(u_{0} \rho_{0}\right)}{H_{m}^{(2)}\left(u_{0} \rho_{0}\right)}\right]
$$

yield an integral which is a known tabulated function. Indeed, the cylinder functions which are solutions to the exact boundary value problem when taken as factors of $\beta_{m}\left(h, \rho^{\prime}\right)$ do not all yield known functions for the $\rho^{\prime}$ integration in (53). Considerable investigation of the $\rho^{\prime}$ integration has been made. The net result of this investigation is that the integration over $\rho^{\prime}$ yields a slowly varying function in $\theta$ that represents a small addition to the free space field. It was expected that this small perturbation would be the extent of the pattern degradation within the critical angles, but the fields computed for the single layer case indicate more significant perturbations on the field configuration than can be attributed to the terms which result from the $\rho^{\prime}$ integration. In general, the $\rho^{\prime}$ integration will yield more significant perturbations as the thickness of the sheath increases. This fact may be gathered intuitively by considering the equivalent antenna aperture size represented by the sheath thickness and an image current sheath in the cylinder.

The perturbations which are evident in figure 8 are attributable to the presence of leaky waves that propagate along the cylinder and sheath and give rise to highly directive radiation patterns. A leaky wave excited at $z^{\prime}=z^{\prime \prime}$ and traveling outward along the cylinder with propagation constant $\nu_{i}$ and attenuation constant $\gamma_{j}$ in the form

$$
\left[\cos \phi^{\prime} E_{\phi^{\prime}}+\sin \phi^{\prime} E_{\rho^{\prime}}\right]=f\left(\rho^{\prime}\right) e^{i\left(\nu_{j}+i \gamma_{j}\right)\left|z^{\prime}-z^{\prime \prime}\right|}
$$

will radiate a directive field with the beam pointing direction given by

$$
\cos \theta= \pm \frac{\nu_{j}}{k_{0}}
$$

and with the beam width determined by $\frac{\gamma_{j}}{k_{0}}$ as will be indicated in the following paragraph.

To compute the field radiated by the leaky waves, consider the beta function for such waves when the sheath is uniform in the $z^{\prime}$ direction

$$
\beta_{m}\left(h, \rho^{\prime}\right)=\sum_{j} \frac{f_{m_{j}}\left(\rho^{\prime}\right)}{2 \pi}\left\{\frac{1}{-\gamma_{j}+i\left(h+\nu_{j}\right)}+\frac{1}{-\gamma_{j}+i\left(h-\nu_{j}\right)}\right\}
$$

where the radial dependence of the function

$$
\left[\cos \phi^{\prime} E_{\phi^{\prime}}+\sin \phi^{\prime} E_{\rho^{\prime}}\right]
$$


for the leaky waves is included in the function $f_{m_{j}}\left(\rho^{\prime}\right)$ and the index $j$ is subscripted to the $m$ to indicate the various leaky waves in each mode. On substituting (56) into (53) there results

$$
\begin{aligned}
E_{\phi_{\text {plasma }}}=-2 \frac{e^{-i k R}}{R} \sum_{i, m} & \left\{\frac{1}{-\gamma_{j}+i\left(h+\nu_{j}\right)}+\frac{1}{-\gamma_{j}+i\left(h-\nu_{j}\right)}\right\}_{h=k_{0} \cos \theta} \\
\cdot & \left\{\int_{0}^{\infty} \rho^{\prime} d \rho^{\prime} f_{m_{j}}\left(\rho^{\prime}\right) e^{i m_{j} \frac{\pi}{2}}\left[J_{m_{j}}\left(u_{0} \rho^{\prime}\right)-H_{m}^{(2)}\left(u_{0} \rho^{\prime}\right) \frac{J_{m}\left(u_{0} \rho_{0}\right)}{H_{m}^{(2)}\left(u_{0} \rho_{0}\right)}\right]\right\}_{h=k_{0} \cos \theta} .
\end{aligned}
$$

The integral within the second braces is generally a slowly varying function of $\theta$ as was discussed above. The integration over $z^{\prime \prime}$ which is implicit in the second bracket in (57) and yields the field pattern of a linear array may negate the above statement, but only the cylinder factor, which is the field due to a point source, is considered here.

The first bracket in (57) yields the rapidly varying dependence of the field on $\theta$. The first term reaches peak magnitude at

and the second term at

$$
h=k_{0} \cos \theta=-\nu_{j}
$$

$$
h=k_{0} \cos \theta=\nu_{j}
$$

which are at symmetrical points about broadside. It will be recalled that the field in (57) is to be linearly added to the free space field in this approximation so that the real and imaginary parts of the rapidly varying functions in (57) are of interest. Figure 9 is a sketch of the real and imaoinary parts of

near

$$
\frac{-1}{\left[-\gamma_{j}+i\left(h-\nu_{j}\right)\right]}
$$

$$
\theta=\cos ^{-1} \frac{\nu_{j}}{k_{0}}
$$

Examination of figure 8 will indicate that the perturbations on the free space field can be accounted for by the complex addition of functions of the type shown in figure 9 to the free space field.

The magnitude of the leaky waves may be approximated with reasonable accuracy from figures 8 a through $8 \mathrm{~g}$ by considering that the peak or null of each sharp field perturbation is the beam pointing direction of the field radiated by the $j$ th leaky wave. The propagation constants, $\nu_{j}$, and the attenuation constants, $\gamma_{j}$, can also be approximated using the peaks

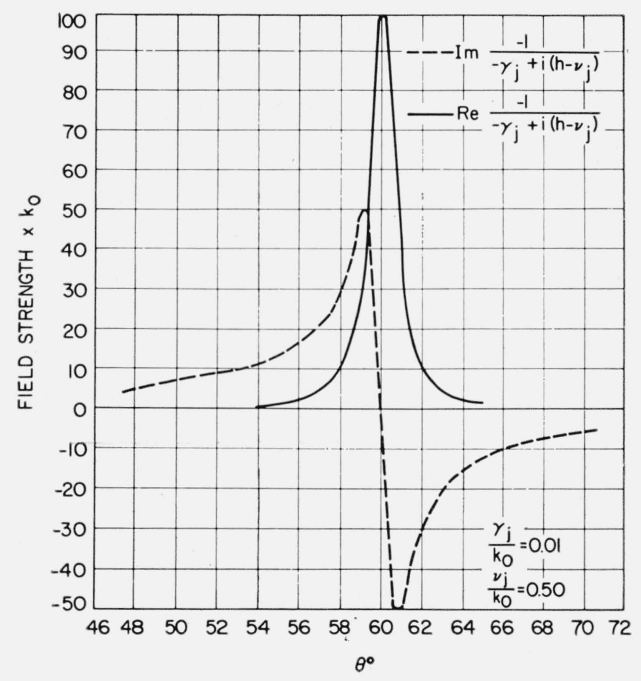

Figure 9. Sketch of the real and imaginary part of a field radiated by leaky waves for small angles near $\mathrm{h}=\nu_{\mathrm{j}}$. 
and, in addition, the widths of the perturbations. The analytical determination of $\nu_{j}$ and $\gamma_{j}$ necessitates the solution of an extremely complicated transcendental equation which involves the cylinder functions. This latter method has not been carried out, but it is suggested that the approximations represent a useful method by which these eigenvalues can be obtained.

Without an analytical expression for the propagation and attenuation constant of the leaky waves, predictions of the effect on the radiation pattern of non-zero losses in the plasma and a finite length of plasma are difficult. One might expect, however, that non-zero losses in the plasma would cause an increase in the attenuation factors $\gamma_{j}$ which (57) indicates would mean a broadening and lowering of the perturbations on the free space field. Similarly, limiting the sheath to finite length would alter the $\beta_{m}\left(h, \rho^{\prime}\right)$ function in a form equivalent to the decrease in aperture size in the $z$ direction and introduce a source field at the discontinuities. Both of these factors would tend to smooth out the field perturbations. The field pattern in the presence of a hypersonic plasma sheath would thus not be expected to have the sharp perturbations of this simplified model.

\section{Conclusions}

It has been demonstrated that quite a significant distortion of the field pattern is effected by the presence of a lossless plasma sheath. The chief characteristics of this distortion are:

1. The presence of critical angles in the field which reduces the power radiated in certain directions so that a blackout zone is introduced in which the field probably is extremely small.

2. The presence of narrow but large peaks and valleys in the field between the critical angles which are attributable to the generation of leaky waves that travel along the cylindrical structure.

3. The generation of a transverse field that changes the plane polarized radiation field when no plasma is present to an elliptically polarized field in every direction except the planes $\phi=0$ and $\phi=\pi$.

4. The presence of slowly varying perturbations on the field which are on the order of several $\mathrm{db}$ due to the non-leaky wave fields in the plasma.

The effect of these distortions on the total field pattern depends on the aperture distribution. In (35) this dependency is made evident: the total field is indicated to be the product of the element, array, and cylinder factors. Only the cylinder factor is shown in the curves. For array factors which yield narrow, broadside beams with very low sidelobes, the distortion under the parameter values discussed in this paper will be represented by increase or decrease of only several $\mathrm{db}$ in pattern gain of the antenna. When the beam is scanned beyond a few degrees, however, or when the array factor is a broad beam, a great deal of distortion occurs for even modest electron densitites in the sheath.

The author is indebted to Dr. A. T. Villeneuve for his many contributions to this paper. The author also wishes to express his appreciation to Dr. W. H. Kummer for suggesting the problem and for a continued interest in and encouragment of the work.

\section{References}

Copson, E. T. (1946), Department of Scientific Research and Experiment, Admiralty Computing Service, No. 106, pp. 15, 16 (The Admiralty, London, England).

Silver, S. (1949), Microwave Antenna Theory and Design, ch. 5, pp. 129-167 (McGraw-Hill Book Co., Inc., New York, N.Y.).

Sommerfeld, A. (1949), Partial Differential Equations in Physics, pp. 88, 92 (Academic Press).

Sonine, N. (1880), Recherches sur les fonctions cylindriques et le developpement des fonctions continues en series, Mathematische Annalen XVI, 30-33.

Wait, J. R. (1959), Electromagnetic Radiation from Cylindrical Structures, (a) ch. 16, (b) page 16, (c) page 29, (d) eq (52) (Pergamon Press, New York and London).

(Paper 67D6-301) 